\title{
Cerebral Hyperperfusion Syndrome after Carotid Revascularization: A Brief Review
}

\author{
Saurabh Anand ${ }^{1} \quad$ Asish K. Sahoo ${ }^{1}$ \\ ${ }^{1}$ Neuroanaesthesia and Neurocritical Care, Artemis Hospital, \\ Gurugram, India
}

J Neuroanaesthesiol Crit Care 2019;6:292-298

\begin{abstract}
Address for correspondence Saurabh Anand, MD (Anaesthesia), Neuroanaesthesia and Neurocritical Care, Artemis Hospital, Gurugram 122001, India (e-mail: saurabhanand03@gmail.com).
\end{abstract}

\author{
Abstract \\ Keywords \\ - carotid artery stenting \\ - carotid \\ endarterectomy \\ - cerebral \\ hyperperfusion \\ - transcranial Doppler
}

Cerebral hyperperfusion (CHS) syndrome is a relatively rare but potentially devastating event that can complicate carotid endarterectomy and carotid stenting. It is associated with increased cerebral perfusion usually more than $100 \%$ from the baseline along with ipsilateral headache, seizures, focal neurological deficits, encephalopathy, intracranial hemorrhage, or subarachnoid hemorrhage. Various risk factors have been identified but most important risk factor is preprocedure evidence of reduced cerebral vasoreactivity with or without contralateral severe carotid stenosis or occlusion. Although diagnosis is suspected in patients with clinical suspicion, it can be radiologically demonstrated with computed tomography (CT), magnetic resonance imaging (MRI), and by dynamic imaging of cerebral perfusion such as transcranial Doppler (TCD), CT, and MR perfusion, and single-photon emission computed tomography (SPECT). Management is usually centered around prompt recognition and active regulation of blood pressure in perioperative and postoperative periods to limit the rise of cerebral blood flow. Prognosis depends on the early detection and prompt management of CHS. If detected early, coupled with intensive blood pressure management, almost all patients will recover over a period of time. For those patients who are diagnosed late and those progressing to intracranial hemorrhage $(\mathrm{ICH})$, the prognosis is not nearly as good.

\section{Introduction}

Carotid endarterectomy (CEA) and carotid artery stenting (CAS) are regarded as treatment modalities for prevention of primary and secondary strokes in patients with significant carotid artery disease. Cerebral hyperperfusion syndrome (CHS) is a relatively rare but potentially devastating event that can complicate both techniques. ${ }^{1}$ It has also been reported in patients of acute stroke treated with intravenous thrombolysis and patients undergoing extracranial to intracranial arterial bypass procedures or cardiothoracic surgery for aortic stenosis. ${ }^{2}$

CHS is generally recognized as a clinical syndrome of ipsilateral headache, seizures, focal neurological deficits, encephalopathy, ICH, or subarachnoid hemorrhage due to regional cerebral hyperperfusion. It is seen more commonly in patients with preprocedure evidence of reduced cerebral vasoreactivity with or without contralateral severe carotid stenosis or occlusion. ${ }^{3,4}$ Although diagnosis

received

August 1, 2019 accepted after revision

September 4, 2019

published online

November 22, 2019 is suspected in patients with clinical suspicion, it can be radiologically demonstrated with computed tomography (CT), magnetic resonance imaging (MRI), and by dynamic imaging of cerebral perfusion such as transcranial Doppler (TCD), CT and MR perfusion, and single-photon emission computed tomography (SPECT). ${ }^{4}$ Although most patients have mild symptoms and signs, progression to severe and life-threatening symptoms can occur if CHS is not recognized and treated adequately. So, prompt recognition and management, typically with acute blood pressure (BP) lowering, is imperative to reduce long-term neurological morbidity. In this brief review, we will be discussing the history, epidemiology, pathophysiology, clinical and radiographic presentation, diagnosis of CHS, and proposed treatment strategies. We will also try to highlight few red flag signs during the preanesthetic evaluation, which should alarm a neuroanesthetist for the possibility of CHS after carotid revascularization.
Copyright @2019 Indian Society of Neuroanaesthesiology and Critical Care
License terms

10.1055/s-0039-1698609 ISSN 2348-0548.
()ㅜ(1) $\odot \circledast$ 


\section{History}

The term normal perfusion pressure breakthrough was coined by Spetzler et al in 1978 to describe cerebral edema and hemorrhage in a region of impaired cerebral autoregulation following arteriovenous malformation resection due to impaired cerebral autoregulation. ${ }^{5}$ Later on, in 1981, Sundt et al found significant postoperative increase in cerebral blood flow (CBF) after CEA and described it as CHS as an explanation to combination of increased arterial blood pressure with the clinical triad of ipsilateral migraine-like headache, seizure, and transient focal neurologic deficits in the absence of cerebral ischemia. ${ }^{6}$

\section{Definition and Epidemiology}

Various radiographic and clinical definitions of CHS have been used in the literature, from purely clinical signs and symptoms in the absence of new postprocedure ischemic stroke to a combination of both clinical changes and hyperperfusion on neuroimaging. Although there is some increase in $\mathrm{CBF}(20-40 \%)$ immediately after the procedure but it is usually self-limiting and subsides within few hours without any symptoms. In some patients CBF increases by more than $100 \%$ compared with baseline values after carotid revascularization, which is often seen within 3 days after revascularization and fall to steady state within 6 to 7 days. It may, however, persist longer till 4 weeks. So, CHS is usually defined as an increase in $\mathrm{CBF}>100 \%$ of baseline along with clinical signs and symptoms. ${ }^{7}$ Rarely, it may develop in patients with increases in perfusion less than $100 \%$ compared with those with baseline values.

Bouri et al in their meta-analysis proposed that the following four criteria be fulfilled for diagnosis of CHS: (1) within 30 days post-CEA; (2) evidence of hyperperfusion (on TCD, SPECT, or CT/MR perfusion imaging) or systolic BP $>180 \mathrm{~mm}$ $\mathrm{Hg}$; (3) clinical features such as new headache, seizure, hemiparesis, Glasgow Coma Scale (GCS) $<15$ or radiological features such as cerebral edema or ICH; and (4) no evidence of new cerebral ischemia, postoperative carotid occlusion, and metabolic or pharmacologic cause. ${ }^{8}$

The true incidence of CHS is difficult to ascertain because of various alterations in the clinical and radiographic diagnosis of CHS in the literature but the incidence of hyperperfusion after carotid revascularization has been estimated between 0.18 and $18.9 \%$. In a meta-analysis of 36 studies by Bouri et al, the incidence of CHS was $1 \%$, and of ICH $0.5 \%$, with a mortality rate of $51 \%$ and permanent disability of $28 \%, 4,9$

\section{Pathophysiology}

Exact pathophysiology of CHS is unclear; it mostly seems to be multifactorial. Additionally, CHS and reperfusion injury may be two pathophysiologically different but interconnected causes of clinical deterioration after revascularization. So, the term CHS has often been used interchangeably with cerebral reperfusion injury and some authors argue that the latter term is more appropriate.
Various proposed mechanisms are:

\section{Impaired Cerebral Autoregulation}

Cerebral autoregulation is the capacity of the cerebral circulation to maintain a constant CBF over a wide range of change in mean arterial pressure $(50-150 \mathrm{~mm} \mathrm{Hg}$ ), which is usually disrupted in carotid artery disease. So, any increases in cerebral blood flow after carotid endarterectomy are not counteracted by paralysis of cerebral autoregulatory mechanisms. In patients with a carotid arterial stenosis, there is regional maximal arteriolar vasodilatation distal to stenosis, which maintains the CBF. In these regions of arteriolar vasodilation, there is reduced cerebral vasoreactivity to $\mathrm{CO}_{2}$ (percentage rise in blood flow velocity in the middle cerebral artery to increased $\mathrm{CO}_{2}$ ), so restoration of perfusion after revascularization may lead to regional hyperemia, which can overwhelm cerebral autoregulatory mechanisms in areas of chronically reduced cerebral vasoreactivity, causing disruption of the blood-brain barrier (BBB) and precipitate CHS. ${ }^{10-12}$

\section{Chronic Hypertension and Related Vascular Changes}

Preoperative longstanding hypertension leads to endothelial dysfunction and microangiopathy which can result in a breakdown of the BBB. Bernstein et al found that in the postmortem study of a patient suspected of death due to CHS, the small arteries and arterioles of the left cerebral cortex showed reactive edema and hyperplasia of endothelial cells, extravasation of erythrocytes, and fibrinoid necrosis. These features of altered vascular pathology are similar to those seen in the brain with malignant hypertension. ${ }^{10}$ Damage to the BBB allows extravasation of toxins and edema into the brain parenchyma, leading to consequent changes. Animal studies show that there might be a role of transforming growth factor beta (TGF 3 ) signaling pathway. ${ }^{13-15}$

\section{Role of Nitric Oxide and Free Radicals}

A possible mediator of impaired autoregulation in CHS is nitric oxide (NO) along with other oxygen-derived free radicals, which causes vasodilatation and can increase the permeability of cerebral vessels in addition to direct toxicity of neurons. ${ }^{16}$ Animal studies have shown that high concentration of NO produced by nitric oxide synthase isoforms are responsible for neuronal injury and subsequent CHS. These mediators are related to reperfusion injury and can persist up to 48 hours post revascularization. ${ }^{17}$

\section{Baroreceptor Dysfunction}

Baroreflex failure is due to dysfunction of baroreceptors after carotid revascularization, which may lead to CHS. So, the buffering action of baroreceptors to the rise in blood pressure is impaired. It may cause a progressive increase in the blood pressure after CEA, which is challenging to control even with blood pressure-lowering therapy..$^{18}$ Therefore, contralateral CEA performed within 3 months of CEA on the other side increases the risk of CHS. Also, the stimulation during endovascular procedure via a balloon or carotid stent results in bradycardia and hypotension, which sometimes persist for longer duration, leading to cerebral ischemia making it prone to early development of $\mathrm{CHS}^{19}$ 


\section{Risk Factors}

Various risk factors have been described but the most important risk factor is diminished cerebrovascular reserve along with postoperative hyperperfusion and hypertension lasting for several hours. Other systemic conditions such as diabetes mellitus (DM), long standing hypertension, old age, contralateral CAS/CEA < 3 months, and high-grade carotid stenosis, may relate to accelerated atherosclerosis, which may lead to carotid stenosis ${ }^{3,4,9,20-23}$ ( - Table 1).

\section{Diagnosis}

\section{Clinical Signs and Symptoms}

The clinical presentation of CHS combines symptoms resulting from brain damage caused by vasogenic edema or symptoms resulting from $\mathrm{ICH}$. Ogasawara et al suggested that the onset of CHS peaks on the sixth postoperative day in patients who undergo CEA and within 12 hours after surgery in those who undergo CAS. ${ }^{20}$ This may be due to the fact that postoperative ischemic cerebral lesions due to emboli are more frequent after CAS than during CEA. Following the emboli resorption and the artery recanalization, cerebral hyperperfusion can occur leading to hemorrhagic transformation in an unviable cerebral area. ${ }^{9}$ Additionally, carotid baroreceptor stimulation during CAS via a balloon or a carotid stent induces transient, sometimes prolonged bradycardia and hypotension that can result in more intense cerebral ischemia than during clamping of the ICA in CEA. Furthermore, subsequent rebound arterial hypertension may induce delayed cerebral hyperperfusion., ${ }^{7,22,23} \mathrm{CHS}$ most commonly presents with ipsilateral pulsating headache, seizure, focal neurological deficits, nausea, or encephalopathy. Headache was the most frequent presenting symptom (30-60\%). Incidence of seizure was approximately 36\% and that of new focal neurological deficits was approximately $31 \%$. Also, some patients develop post procedure cognitive impairment, leading to neuropsychological dysfunctions after 3 days of procedure. Routine use of antiplatelets in CAS leads to somewhat higher incidence of ICH in CAS than CEA. ${ }^{9}$

\section{Neuroimaging}

Diagnosis of CHS is based on two factors: Demonstration of impaired cerebrovascular reserve preoperatively and demonstration of hyperperfusion in the postoperative period. Multiple imaging modalities are available to identify patients suffering from CHS or at risk for CHS. Few commonly used modalities are transcranial Doppler (TCD), computerized tomography (CT),

Table 1 Red flags in preanesthetic evaluation

\footnotetext{
- Diabetes mellitus (especially if uncontrolled)

- Longstanding hypertension

- Stroke

- Recent (<3 months) contralateral carotid endarterectomy

- High-grade carotid artery stenosis

- Impaired VMR

- Contralateral carotid stenosis
}

magnetic resonance imaging (MRI), MR perfusion (MRP), and single-photon-emission CT (SPECT).

\section{Transcranial Doppler}

Transcranial Doppler is a noninvasive and real-time bedside modality, which can determine impaired cerebrovascular reserve (CVR) and hyperperfusion. TCD measures cerebral blood flow velocity in the middle cerebral artery with a Doppler probe through a transcranial bone window. In CHS, TCD typically shows a 150 to $300 \%$ increase in the ipsilateral middle-cerebral-artery flow velocity, and normalization of hyperperfusion with blood pressure reduc-

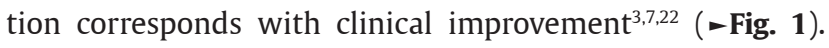
Preoperatively, CVR can be calculated to determine the patients who are at risk for developing CHS. Buczek et al calculated CVR as $\left(\mathrm{V}_{\max }-\mathrm{V}_{\mathrm{o}}\right) / \mathrm{V}_{\mathrm{o}} \times 100 \%$, where $\mathrm{V}_{\max }$ is the maximum increase of mean velocity (MV) in the MCA recorded every $5 \mathrm{~min}$ for $30 \mathrm{~min}$ after intravenous administration of $1 \mathrm{~g}$ acetazolamide, and Vo is the middle cerebral artery (MCA) baseline MV. ${ }^{24}$ They used values $<25 \%$ as definition for impaired CVR. Markus et al described breath-holding index (BHI) as an indicator for deranged CVR. MCA velocities are calculated after 30 seconds of voluntary breadth holding. Then BHI is calculated as $\left(V_{\text {end }}-V_{\text {baseline }}\right) / V_{\text {baseline }} \times 100 /$ seconds of breadth holding. BHI of $<0.69$ is considered to represent an impaired CVR. ${ }^{25} \mathrm{~A}$ decreased $\mathrm{BHI}$ represents failure of the collateral flow to maintain adequate cerebral perfusion in response to the hypercapnic challenge. Other TCD criteria for the prediction of postoperative hyperperfusion in patients with recent CEA or CAS are increase in peak blood flow velocity or mean flow velocity $>100 \%$ after recanalization of artery. ${ }^{26,27}$ The limitations of TCD are that it is operator dependent, 10 to $15 \%$ patients have difficult insonation, and there might be variations in circle of Willis causing difficulty in accurate interpretation. So, incorporating the use of TCD in preanesthetic evaluation to check for impaired CVR can help a neuroanasthetist to assess the risk for developing CHS in perioperative period.

\section{Computerized Tomography}

Computerized tomography scan is not a specific diagnostic modality for detecting CHS but it can detect postoperative bleed, cerebral edema, or mass effect. So, it should be used as initial imaging technique if there is some suspicion of $\mathrm{CHS}^{3}$

\section{Single-Photon Emission CT}

Single-photon emission CT can detect alterations in brain perfusion and the impairment of preoperative cerebrovascular reactivity (after acetazolamide). A diffuse asymmetric pattern of preoperative $\mathrm{CBF}$ reduction seems to be characteristic in these patients. ${ }^{28,29}$ Ogasawara et al suggested that hyperperfusion for first 3 postoperative days on SPECT predisposes to CHS development. ${ }^{20}$

\section{Magnetic Resonance Techniques}

Magnetic resonance imaging abnormalities can be similar to those seen on CT of the brain including white matter edema, 


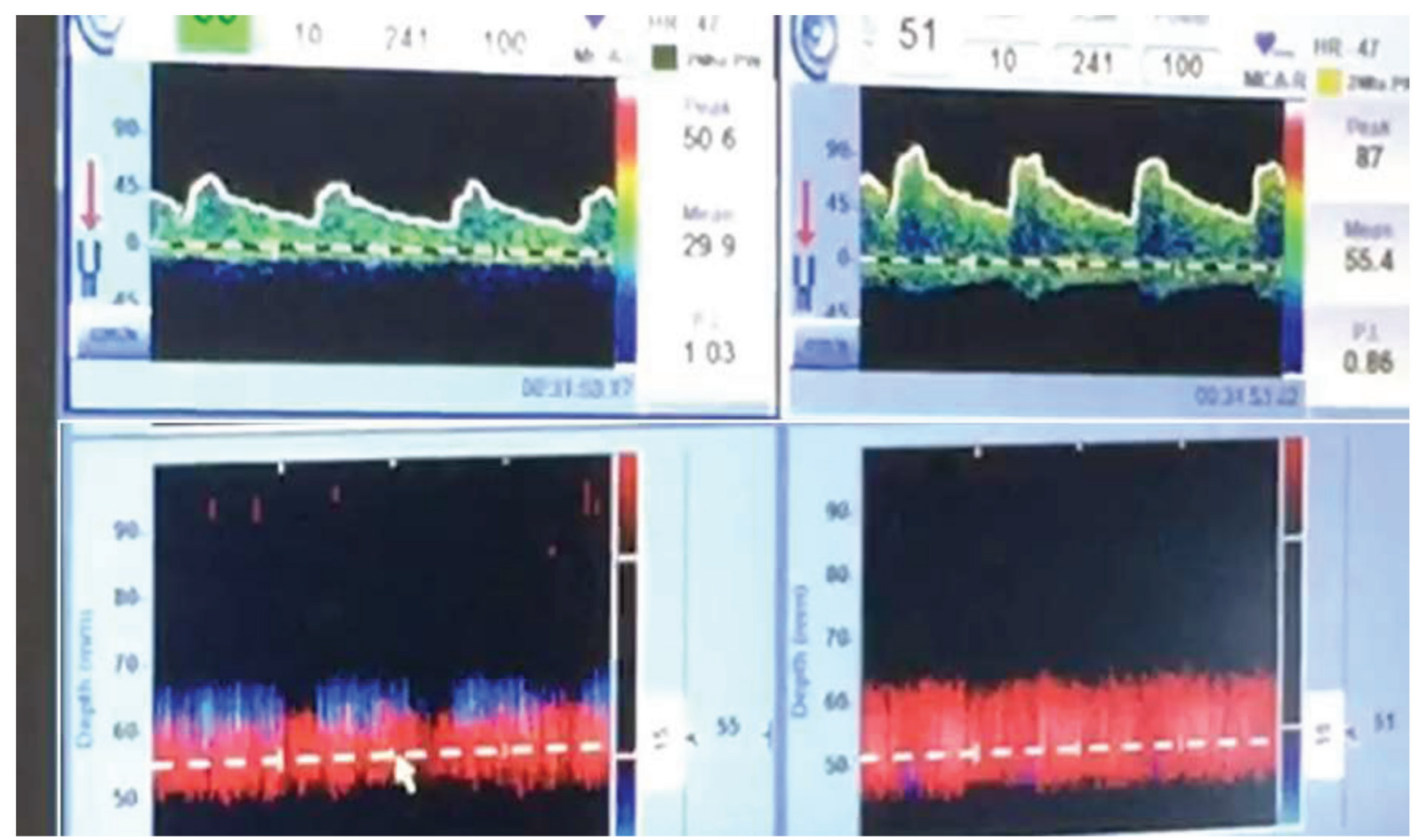

Fig. 1 Transcranial Doppler of a patient with carotid stenosis who underwent CAS. Baseline MCA velocity (left) became almost double after 12 hours of CAS. Patient was actively managed with intensive BP control for 48 hours and was discharged when the velocities returned to baseline values.

focal infarction, and hemorrhage. On noncontrast brain MRI, T2 or FLAIR sequences may show focal, unilateral, or more diffuse hyperintensities consistent with vasogenic edema, and perfusion-weighted MRI may show a relative hyperperfusion in the revascularized hemisphere. ${ }^{30}$ MR perfusion images show a relative interhemispheric CBF differences in patients with CHS after CEA. Arterial spin labeling MRI can also be used to detect cerebral hyperperfusion and has been identified as a tool to predict risk for hemorrhagic transformation after ischemic stroke reperfusion. ${ }^{31,32}$

\section{Ocular Pneumoplethysmography}

Postoperative increase of ocular blood flow greater than $204 \%$ is associated with a high risk for $\mathrm{CHS}^{33}$

\section{Transcranial Color-Coded Real-Time Ultrasonography with Echo Contrast Agents}

A 1.5-fold postoperative increase of MCA mean flow velocity within 4 days compared with preoperative levels yielded high accuracy predictions of $\mathrm{CHS}^{34}$

\section{Transcranial Regional Cerebral-Oxygen-Saturation Monitoring}

An increase in regional cerebral oxygen saturation is a sign of increase in the cerebral blood flow when cerebral oxygen consumption and arterial oxygen saturation are stable. It can be estimated by near-infrared spectroscopy. ${ }^{35}$

\section{Electroencephalography}

Although it is not specific but some cases show periodic lateralized epileptiform discharges, even in the absence of seizures or post seizure. These discharges are indicative of localized cerebral foci of irritability, which may not correspond to higher risk for CHS. ${ }^{3,36}$

\section{Management Strategies}

\section{Prevention and Treatment}

Preventive strategies for CHS include proper blood pressure control in the perioperative period, and consideration of timing of surgery, type of anesthesia, and use of free radical scavengers. There are no data from randomized trials comparing the optimal perioperative management protocol for patients with CHS, due to the rarity of this complication. There are no data regarding the superiority of CAS over CEA or vice versa and the differences between CAS and CEA with respect to CHS are illustrated in - Table 2 .

\section{Blood Pressure Control}

Although it is recommended to maintain "normotension" after CEA or CAS, there is lack of data regarding target BP to prevent CHS after intracranial thrombectomy. During carotid intervention by both CEA and CAS, intraprocedural hypotension can occur, because of denervation of carotid baroreceptors, followed by a rebound hypertension; this is more so after CAS. So, intensive perioperative monitoring of BP, preferably by invasive methods, should be done and such patients should be monitored in a specialized unit. Most of the authors recommend to keep SBP $<120-140 \mathrm{~mm} \mathrm{Hg}$ as seldom CHS develop with SBP $<135 \mathrm{~mm} \mathrm{Hg.,7,9,23}$ Bouri et al found that the cumulative incidence of CHS increased above 
Table 2 Difference between CAS and CEA with respect to CHS

- Postoperative ischemic cerebral lesions due to emboli are more frequent after CAS than during CEA

- Following the emboli resorption and the artery recanalization, cerebral hyperperfusion can occur, leading to hemorrhagic transformation in an unviable cerebral area

- Carotid baroreceptor stimulation during CAS via a balloon or a carotid stent induces transient, sometimes prolonged bradycardia and hypotension that can result in more intense cerebral ischemia than during clamping of the ICA in CEA

- Cerebral hyperperfusion occur earlier after CAS then CEA

an inflection point of postoperative systolic BP $>150 \mathrm{~mm}$ Hg. ${ }^{8}$ There are no randomized prospective studies on optimal antihypertensive medications in management of CHS after CEA or CAS. Beta-blockers, such as labetalol, are a good first-line agent, as they decrease cerebral perfusion pressure and MAP and do not directly affect cerebral blood flow. Clonidine is also used after CEA for its sympatholytic properties. Labetalol, which has a mixed alfa- and beta-adrenergic antagonistic action with no effect on $\mathrm{CBF}$ and decreases the MAP by $30 \%$ has been successfully used in CHS. Due to their vasodilatory properties and potential to increase cerebral blood flow, it is generally advised to avoid sodium nitroprusside, nitrates, hydralazine, ACE-inhibitors, and calcium channel blockers acutely. ${ }^{3}$ Although there are limited data on the duration of therapy, treatment should be continued until cerebral autoregulation is restored. The time period for this varies between patients. Some recommend treatment for 6 months, whereas others use equalization of TCD signals in both the hemispheres to guide the duration of treatment. It is important that patients are not discharged with severe hypertension or a SBP that is rising. Patients with labile BP should be considered for a home BP monitor for the first postoperative week, after review by a physician and should refer to the treating physician if SBP > $160 \mathrm{~mm} \mathrm{Hg}$.

\section{Timing of Surgery}

As per the American Heart Association and American Stroke Association Guidelines, the best benefits are obtained within 2 weeks of the ischemic stroke or transient ischemic attack. However, there is a potential risk of CHS and ICH if surgery is done early in patients with large cerebral infarction or stroke in evolution. ${ }^{37}$ Moreover, in the case of bilateral carotid stenosis, the risk of CHS is higher in a patient who undergoes CEA in less than 3 months of the initial procedure on the contralateral side. These factors should be considered when planning CEA. ${ }^{14,38}$

\section{Type of Anesthesia}

High doses of halogenated inhalation anesthetic agents may increase CBF, which may predispose to CHS. ${ }^{3}$ So, titrated dose of volatile anesthetic agents should be used. Propofol have minimal effects on the CBF; therefore, it is a safer option in these patients. There is not enough evidence from randomized trials comparing carotid endarterectomy under local anesthetics with carotid endarterectomy under general anesthetics. $^{39}$

\section{Antiepileptic Medications}

There are no available data recommending prophylactic use of anticonvulsant therapy in patients undergoing carotid revascularization. However, in the presence of seizures, treatment with anticonvulsants is always indicated. ${ }^{3}$

\section{Treatment of Cerebral Edema}

There is no indication for the prophylactic treatment of cerebral edema in CHS. However, if the cerebral edema progresses to a point where it is causing uncontrollable increases in intracranial pressure, the use of sedation, osmotic agents (mannitol, hypertonic saline), and ultimately mechanical ventilation may be necessary. ${ }^{14}$

\section{Anticoagulation and Antiplatelet Therapy}

Seizures after carotid endarterectomy are a contraindication for anticoagulation therapy. Although not definitively linked to an increased incidence of CHS, it does seem to be linked to ICH. ${ }^{21}$ Management of ICH after CAS is more complicated as the patient is usually on dual antiplatelets for the prevention of stent thrombosis. In such cases, patient might be shifted to single antiplatelet, which might further increase the risk of stent thrombosis. No guideline is available for management of such cases. Recently published PATCH trial did recommend not to use platelet transfusion for the management of ICH. ${ }^{40}$

\section{Role of Antioxidants}

In a study by Ogasawara et al, pretreatment with edaravone, a free radical scavenger, decreased the incidence of hyperperfusion after CEA when measured by SPECT. ${ }^{41}$ The evidence for the use of antioxidants and free radical scavengers is limited; therefore, larger studies are required to assess its usefulness.

\section{Prognosis}

Prognosis depends on the early detection and prompt management of CHS. If detected early, coupled with intensive blood pressure management, almost all patients will recover over a period of time. For those patients who are diagnosed late and those progressing to $\mathrm{ICH}$, the prognosis is not nearly as good, with up to $30 \%$ remaining partially disabled and with mortality rates up to $50 \% .^{21,42,43}$ Thus, although intracerebral hemorrhage in CHS is rare, it is almost uniformly a devastating occurrence.

\section{Conclusion}

CHS is a rare but serious complication after carotid revascularization. Early identification of at-risk individuals, particularly with impaired CVR by TCD or other radiological modalities, can prepare the physician to deal with it. Aggressive blood pressure management has shown to be greatly beneficial in preventing morbidity and mortality associated 
Table 3 Institute protocol for detection and management of CAS

1. Preoperative history and risk factors to be seen in detail

- Diabetes mellitus

- Longstanding hypertension

- Stroke

- Recent (<3 months) contralateral carotid revascularization procedure

- High-grade carotid artery stenosis

2. Preoperative transcranial Doppler (TCD) in every patient

- Baseline MFV in both MCA noted; along with that a note is made about the delayed systolic acceleration or the blunted waveform on the affected side

- VMR assessed by BHI and graded as absent or insufficient if the values are less than 0.3 and 0.69 , respectively. If the value is more than 0.69 , it is termed as preserved VMR

- Intraoperatively, invasive blood pressure monitoring is done in every case, which is continued postoperatively

\section{Postoperative TCD}

- Done in every patient just after the completion of the procedure and two times daily for first 48 hours. Any increase in MFV in TCD (ipsilateral side) is noted

- If increase in MFV is more than $100 \%$, then strict control of BP is done with labetalol, as first-line drug, started as infusion dose of maximum up to $1 \mathrm{mg} / \mathrm{min}$. Along with that, oral antihypertensive, preferably clonidine, is also started

- Intensive care unit stay is prolonged for 48 hours at least. Regular TCD is also performed to see that BP control has resulted in stabilizing the MFV of MCA. In the first 2 weeks, a strict control of BP is advised. Home-based blood pressure monitoring every 4 hours is recommended, if discharged. If more than 2 consecutive readings are higher than the set target or severe headache, then the patient is advised to contact the hospital

- If there is ipsilateral headache and radiological signs of cerebral edema, osmotic diuretics and antiepileptic medications are started

- A follow-up TCD is advised in high-risk patients after 1 week

with it. Further research is warranted regarding preprocedure cerebral vasoreactivity testing to predict CHS in patients undergoing CEA and CAS, and in defining optimal postprocedure hemodynamic management to prevent and treat CHS. The management protocol followed in our institute has been illustrated in - Table 3.

\section{Conflict of Interest}

None declared.

\section{References}

1 Yu S, Liebeskind DS, Dua S, et al. UCLA Stroke Investigators. Postischemic hyperperfusion on arterial spin labeled perfusion MRI is linked to hemorrhagic transformation in stroke. J Cereb Blood Flow Metab 2015;35(4):630-637

2 Zhang Y, Kumar A, Tezel JB, Zhou Y. Imaging evidence for cerebral hyperperfusion syndrome after intravenous tissue plasminogen activator for acute ischemic stroke. Case Rep Neurol Med 2016;2016:8725494

3 van Mook WN, Rennenberg RJ, Schurink GW, et al. Cerebral hyperperfusion syndrome. Lancet Neurol 2005;4(12): 877-888

4 Wang GJ, Beck AW, DeMartino RR, Goodney PP, Rockman CB, Fairman RM. Insight into the cerebral hyperperfusion syndrome following carotid endarterectomy from the national Vascular Quality Initiative. J Vasc Surg 2017;65(2):381-389

5 Spetzler RF, Wilson CB, Weinstein P, Mehdorn M, Townsend J, Telles D. Normal perfusion pressure breakthrough theory. Clin Neurosurg 1978;25:651-672

6 Sundt TM, Jr. Sharbrough FW, Piepgras DG, Kearns TP, Messick JM Jr. O'Fallon WM. Correlation of cerebral blood flow and electroencephalographic changes during carotid endarterectomy: with results of surgery and hemodynamics of cerebral ischemia. Mayo Clin Proc 1981;56(9):533-543

7 Kirchoff-Torres KF, Bakradze E. Cerebral hyperperfusion syndrome after carotid revascularization and acute ischemic stroke. Curr Pain Headache Rep 2018;22(4):24

8 Bouri S, Thapar A, Shalhoub J, et al. Hypertension and the post-carotid endarterectomy cerebral hyperperfusion syndrome. Eur J Vasc Endovasc Surg 2011;41(2):229-237

9 Moulakakis KG, Mylonas SN, Sfyroeras GS, Andrikopoulos V. Hyperperfusion syndrome after carotid revascularization. J Vasc Surg 2009;49(4):1060-1068

10 Bernstein M, Fleming JF, Deck JH. Cerebral hyperperfusion after carotid endarterectomy: a cause of cerebral hemorrhage. Neurosurgery 1984;15(1):50-56

11 Benzel EC, Hoppens KD. Factors associated with postoperative hypertension complicating carotid endarterectomy. Acta Neurochir (Wien) 1991;112(1-2):8-12

12 Sakaki T, Tsujimoto S, Nishitani M, Ishida Y, Morimoto T. Perfusion pressure breakthrough threshold of cerebral autoregulation in the chronically ischemic brain: an experimental study in cats. J Neurosurg 1992;76(3):478-485

13 Skydell JL, Machleder HI, Baker JD, Busuttil RW, Moore WS. Incidence and mechanism of post-carotid endarterectomy hypertension. Arch Surg 1987;122(10):1153-1155

14 Farooq MU, Goshgarian C, Min J, Gorelick PB. Pathophysiology and management of reperfusion injury and hyperperfusion syndrome after carotid endarterectomy and carotid artery stenting. Exp Transl Stroke Med 2016;8(1):7

15 Cacheaux LP, Ivens S, David Y, et al. Transcriptome profiling reveals TGF-beta signaling involvement in epileptogenesis. J Neurosci 2009;29(28):8927-8935

16 Janigro D, West GA, Nguyen TS, Winn HR. Regulation of blood-brain barrier endothelial cells by nitric oxide. Circ Res 1994;75(3):528-538

17 Holm J, Nilsson U, Waters N, Waters S, Jonsson O. Production of free radicals measured by spin trapping during operations for stenosis of the carotid artery. Eur J Surg 2001;167(1):4-9

18 Robertson D, Hollister AS, Biaggioni I. Netterville JL, MosquedaGarcia R, Robertson RM. The diagnosis and treatment of baroreflex failure. N Engl J Med 1993;329(20):1449-1455 
19 Timmers HJLM, Wieling W, Karemaker JM, Lenders JWM. Baroreflex failure: a neglected type of secondary hypertension. Neth J Med 2004;62(5):151-155

20 Ogasawara K, Yukawa H, Kobayashi M, et al. Prediction and monitoring of cerebral hyperperfusion after carotid endarterectomy by using single-photon emission computerized tomography scanning. J Neurosurg 2003;99(3):504-510

21 Piepgras DG, Morgan MK, Sundt TM Jr. Yanagihara T, Mussman LM. Intracerebral hemorrhage after carotid endarterectomy. J Neurosurg 1988;68(4):532-536

22 Ogasawara K, Inoue T, Kobayashi M, et al. Cerebral hyperperfusion following carotid endarterectomy: diagnostic utility of intraoperative transcranial Doppler ultrasonography compared with single-photon emission computed tomography study. AJNR Am J Neuroradiol 2005;26(2):252-257

23 Abou-Chebl A, Reginelli J, Bajzer CT, Yadav JS. Intensive treatment of hypertension decreases the risk of hyperperfusion and intracerebral hemorrhage following carotid artery stenting. Catheter Cardiovasc Interv 2007;69(5):690-696

24 Buczek J, Karliński M, Kobayashi A, Białek P, Członkowska A. Hyperperfusion syndrome after carotid endarterectomy and carotid stenting. Cerebrovasc Dis 2013;35(6):531-537

25 Markus HS, Harrison MJ. Estimation of cerebrovascular reactivity using transcranial Doppler, including the use of breath-holding as the vasodilatory stimulus. Stroke 1992;23(5):668-673

26 Jansen C, Sprengers AM, Moll FL, et al. Prediction of intracerebral haemorrhage after carotid endarterectomy by clinical criteria and intraoperative transcranial Doppler monitoring: results of 233 operations. Eur. J Vasc Surg 1994;8(2):220-225

27 Yoshimoto T, Shirasaka T, Yoshizumi T, Fujimoto S, Kaneko $\mathrm{S}$, Kashiwaba T. Evaluation of carotid distal pressure for prevention of hyperperfusion after carotid endarterectomy. Surg Neurol 2005;63(6):554-557, discussion 557-558

28 Hosoda K, Kawaguchi T, Ishii K, et al. Prediction of hyperperfusion after carotid endarterectomy by brain SPECT analysis with semiquantitative statistical mapping method. Stroke 2003;34(5):1187-1193

29 Sfyroeras GS, Karkos CD, Arsos G, et al. Cerebral hyperperfusion after carotid stenting: a transcranial Doppler and SPECT study. Vasc Endovasc Surg 2008

30 Karapanayiotides T, Meuli R, Devuyst G, et al. Postcarotid endarterectomy hyperperfusion or reperfusion syndrome. Stroke 2005;36(1):21-26

31 Ances BM, McGarvey ML, Abrahams JM, et al. Continuous arterial spin labeled perfusion magnetic resonance imaging in patients before and after carotid endarterectomy. J Neuroimaging 2004;14(2):133-138

32 Fukuda T, Ogasawara K, Kobayashi M, et al. Prediction of cerebral hyperperfusion after carotid endarterectomy using cerebral blood volume measured by perfusion-weighted MR imaging compared with single-photon emission CT. AJNR Am J Neuroradiol 2007;28(4):737-742

33 Nicholas GG, Hashemi H, Gee W. Reed JF III. The cerebral hyperperfusion syndrome: diagnostic value of ocular pneumoplethysmography. J Vasc Surg 1993;17(4):690-695

34 Fujimoto S, Toyoda K, Inoue T, et al. Diagnostic impact of transcranial color-coded real-time sonography with echo contrast agents for hyperperfusion syndrome after carotid endarterectomy. Stroke 2004;35(8):1852-1856

35 Pennekamp CW, Immink RV, den Ruijter HM, et al. Near-infrared spectroscopy can predict the onset of cerebral hyperperfusion syndrome after carotid endarterectomy. Cerebrovasc Dis 2012;34(4):314-321

36 Bozkurt MF, Saygi S, Erbas B. SPECT in a patient with postictal PLEDs: is hyperperfusion evidence of electrical seizure? Clin Electroencephalogr 2002;33(4):171-173

37 Kernan WN, Ovbiagele B, Black HR, et al. American Heart Association Stroke Council, Council on Cardiovascular and Stroke Nursing, Council on Clinical Cardiology, and Council on Peripheral Vascular Disease. Guidelines for the prevention of stroke in patients with stroke and transient ischemic attack: a guideline for healthcare professionals from the American Heart Association/American Stroke Association. Stroke 2014;45(7):2160-2236

38 Giordano JM, Trout HH, III. Kozloff L, DePalma RG. Timing of carotid artery endarterectomy after stroke. J Vasc Surg 1985;2(2):250-255

39 Kaisti KK, Langsjo JW, Aalto S, et al. Effects of sevoflurane, propofol, and adjunct nitrous oxide on regional cerebral blood flow, oxygen consumption, and blood volume in humans. Anesthesiology 2003;99(3):603-613

40 Baharoglu MI, Cordonnier C, Salman RA, et al. PATCH Investigators. Platelet transfusion versus standard care after acute stroke due to spontaneous cerebral haemorrhage associated with antiplatelet therapy (PATCH): a randomised, open-label, phase 3 trial. Lancet 2016;387(10038):2605-2613

41 Ogasawara K, Inoue T, Kobayashi M, Endo H, Fukuda T, Ogawa A. Pretreatment with the free radical scavenger edaravone prevents cerebral hyperperfusion after carotid endarterectomy. Neurosurgery 2004;55(5):1060-1067

42 Meyers PM, Higashida RT, Phatouros CC, et al. Cerebral hyperperfusion syndrome after percutaneous transluminal stenting of the craniocervical arteries. Neurosurgery 2000;47(2):335343, discussion 343-345

43 Lieb M, Shah U, Hines GL. Cerebral hyperperfusion syndrome after carotid intervention: a review. Cardiol Rev 2012;20(2):84-89 